\title{
La Nueva Gobernanza de los sistemas universitarios
}

The new gobernance of University Systems

DOI: http://dx.doi.org/10.29105/pgc4.8-1

Barbara, Kehm ${ }^{1}$

Universidad de Glasgow

https://orcid.org/0000-0002-8890-9029

\section{RESUMEN}

El presente artículo es producto de una revisión bibliográfica, cuyo objetivo consistió en estudiar y clasificar los regímenes de gobernanza de las universidades a nivel internacional, sus dinámicas de cambios y tendencias evolutivas, con un enfoque cualitativo, diseño no experimental, bajo un nivel documental-bibliográfico transversal. Tras la revisión documental se encontró que: la gobernanza universitaria es un concepto que tiene una constante evolución, adaptándose a los diversos panoramas y diferentes perspectivas. En conclusión, las políticas educativas responden cada vez más a una arena internacional en las que están sucediendo la configuración de agendas, los desarrollos de criterios y directrices.

Palabras clave: Gestión pública, gobierno, gobernanza, política, universidad..

\section{ABSTRACT}

This article is the product of a bibliographic review, which objective was to study and classify the governance regimes of universities at international level, their dynamics of changes and evolutionary tendencies, with a qualitative approach, non-experimental design, under transversal documentary-bibliographic level. After the documentary review, it was found that: university governance is a concept that has a constant evolution, adapting to different scenarios and different perspectives. In conclusion, educational policies respond more and more to an international arena in which the configuration of agendas, the development of criteria and guidelines are happening.

Key words: Government, governance, management public, politics, university.

Cómo referenciar este artículo:

Kehm, B. (2018). La Nueva Gobernanza de los sistemas universitarios. Revista Política, Globalidad y Ciudadanía, 4(8), 14-19. Recuperado de http://revpoliticas.uanl.mx/index.php/RPGyC/article/view/99

Recibido: 06 de Enero 2018 - Aceptado: 30 de Febrero 2018

(cc) BY-NC-ND

1 Doctora en Literatura Alemana por Universidad Ruhr Bochum. Docente Investigadora en la Escuela de Educación de la Universidad de Glasgow. Email: Barbara.Kehm@glasgow.ac.uk

Revista Política, Globalidad y Ciudadanía, Vol. 4 No. 8, Julio - Diciembre 2018, Universidad Autónoma de Nuevo León, Monterrey, México, ISSN 2395-8448. pp 14-19. http://revpoliticas.uanl.mx/index.php/RPGyC/article/view/99 


\section{INTRODUCCIÓN}

El presente artículo contiene diversas recopilaciones de investigaciones realizada por académicos universitarios europeos, quienes se han desempeñado en el sector universitario y responsables de políticas educativas universitarias, así mismo, el documento cuenta con puntos de vista de Europa y América Latina.

El concepto de gobernanza sirve para explicar los mecanismos de la coordinación de actividades de los distintos, aunque interdependientes, actores en un campo determinado. También se puede describir mecanismos de coordinación y dirección en el marco de los procesos económicos. En pocas palabras, el término gobernanza significa procesos de regulación y coordinación orientados al bienestar, que ayudan a resolver problemas sociales.

La gobernanza universitaria tiene que pasar por un proceso de innovación para poder adaptarse con mayor facilidad, vivimos en un mundo que está en constante cambio y cada día es más dinámico, así que, el sistema universitario ha pasado por una constante evolución en sus diversas ramas de estudio con el fin de adaptarse a las nuevas necesidades.

El desarrollo de nuevas herramientas y enfoques es vital para diseñar nuevos sistemas universitarios, sin embargo, los cambios en la gestión pública son más escasos y difíciles, y no solo porque son los que exigen mayor financiación adicional, sino por su ejecución. Así que hay que crear políticas universitarias que se puedan implementar en todas sus ramas y en la vida diaria de los estudiantes y docentes.

\section{2.- FUNDAMENTO TEÓRICO}

\section{Gobernanza y gestión publica}

La gobernanza y la nueva gestión pública son parte de la agenda europea para modernizar la universidad y hacerla más responsable de las necesidades sociales y económicas en la era de la sociedad del conocimiento. Es un claro ejemplo de cómo se puede implementar exitosamente las políticas públicas, para mejorar la economía y la sociedad de cualquier país, todas las universidades y gobiernos afrentan un gran reto, que es investigar e implementar acciones a favor de los estudiantes, docentes y ciudadanos.

Mucho se habla sobre lo que más se necesita es una idea unificada de la universidad del siglo XXI que pueda establecer una base para renovar la confianza de la sociedad en la institución, sin embargo, lo que más se necesita son nuevas formas de evaluar y enseñar con bases fundamentales para el desarrollo de una mejor sociedad. La innovación se puede dar, teóricamente, en todas y en cada una de sus funciones, en cada una de ellas, el cambio es más fácil o difícil, tiene mayor o menor impacto y requiere más o menos financiación, pero se debe de realizar para poder avanzar.

El libro menciona que la gobernanza a nivel sistémico implica una nueva relación entre universidad, Estado y sociedad, que muy seguido se necesitan cambiar de enfoques y crear una nueva visión y gestión pública; esto me parece excelente ya que su intención principal es que dejen de ser instituciones burocráticas a organizaciones gestionadas y así, crear una mayor participación en el Estado como actores.

Como en todos lados, existen los conflictos de poder, que hasta donde debe de llegar la participación de una universidad y que tipo de esquema organizativo se necesita para alcanzar la excelencia, garantizar la curiosidad libre y demostrar responsabilidad social y cultural.

La nueva gestión pública es la primera narrativa, que vio la luz a finales de los años setenta como parte de las reformas conservadoras de Margaret Thatcher. Esta combina jerarquías y mercados, y, básicamente, 
refuerza el papel de los gestores, además de obligar a las universidades a diversificar las bases sobre las que se fundaron.

La segunda narrativa se centra en la gobernanza en red, que emergió en los años noventa, trata sobre el Estado-Nación y su pérdida de funciones, autoridad y responsabilidad en dominios regionales y supranacionales, la distribución de poder es más difusa y pluralista. Esto obliga a las universidades a operar estratégica y simultáneamente en distintas redes en los campos regional, nacional e internacional.

La tercera narrativa, también llamada Neoweberiana, es sobre revitalización democrática de instituciones públicas altamente burocratizadas. Esto se puede observar en muchos países latinoamericanos, donde se observa una mayor participación en las decisiones institucionales y en las políticas de desarrollo público.

Así que, las narrativas se enfocan mucho en la profesionalización de la gestión pública y en que las instituciones públicas satisfagan las necesidades de la ciudadanía. Con estas tres narrativas y sus implicaciones en la gobernanza universitaria, los autores intentan predecir algunos síntomas e instrumentos para la gestión institucional. También proponen una agenda de investigación adicional, para poder desarrollar sus reformas educativas y planes de estudio de la mano.

Cada vez más, la gobernanza universitaria se entiende como una combinación de relaciones verticales como jerarquías y mercados, y de relaciones horizontales como redes de actores. Las políticas nacionales, se están adaptando a estos nuevos contextos y condiciones, tratando al mismo tiempo de salvaguardar la tradición nacional, sin embargo, se necesitan modernizar todos sus aspectos para poder competir a nivel internacional con otras universidades.

La combinación de diferencias y semejanzas en la gobernanza universitaria se puede demostrar con el modelo de ecualización de la gobernanza, aunque finalmente necesita operar en cada dimensión con una lista de indicadores apropiados, tienen la intención de transformar las actitudes de la profesión académica y la manera en la que tradicionalmente hacen su trabajo en la enseñanza y la investigación. Un factor importante en este desarrollo es la profesionalización y la orientación gestora de los líderes de las universidades. Rectores o presidentes ya no son vistos como los primeros entre iguales, con un poder de decisión relativamente limitado, sino como ambiciosos gerentes de entidades corporativas.

En la actualidad, los universitarios y ciudadanos ya no creen que el gobierno pueda realizar un cambio verdadero en la sociedad, así que existe una presión más fuerte para ejecutar acciones que si funcionen; los ciudadanos cada vez están más involucrados para crear políticas públicas y ser un parteaguas en el gobierno.

El enfoque principal es la cuestión de cómo las universidades, en tanto que organizaciones, reaccionan a cambios de condiciones en su ambiente, como los estudiantes y docentes lograr adaptarse, con qué facilidad, etc. Esto es muy importante para lograr una mayor participación en las políticas públicas y aumentar el rating universitario y político.

Uno de los economistas más citados, Paul E. Samuelson (1954) menciona que para que un bien sea considerado público ha de cumplir dos prerrequisitos: no ser excluyente y no tener rivalidad. Lo primero conlleva que cada uno puede usar o tener acceso a dicho bien sin excepción; mientras que lo segundo significa que el uso del bien por un individuo no debe mermar su disponibilidad para otros usuarios.

En el texto se debate si la educación debe de ser un bien privado o público, ya que recibe financiamiento por parte del gobierno y organizaciones y tienen que seguir con las reformas educativas que implementan los legisladores. En el campo de la educación superior esto va más por el dado de un proceso de privatización y a la introducción de fuerzas mercantiles, por ejemplo, en forma de competencia por la reputación y por la financiación. Con esto, la naturaleza de la universidad se ha acercado más a lo que se entendería por

Revista Política, Globalidad y Ciudadanía, Vol. 4 No. 8, Julio - Diciembre 2018, Universidad Autónoma de Nuevo León, Monterrey, México, ISSN 2395-8448. pp 14-19. http://revpoliticas.uanl.mx/index.php/RPGyC/article/view/99 
un bien privado susceptible de ser comercializado tal como lo prevé el Acuerdo General sobre el Comercio de Servicios.

El escepticismo sobre si la educación aún representa un bien público ha crecido en los últimos treinta años bajo el influjo de políticas neoliberales en las reformas del sector público, sin embargo, se necesita encontrar un balance y ser más transparente en sus políticas universitarias.

Mediante la adopción de nuevas estrategias y modalidades, los gobiernos buscan inducir cambios en la conducción de las universidades: nuevas formas de gobernanza, fijación de prioridades y metas, autoevaluación en función de indicadores de desempeño, foco en la eficiencia interna y externa, atención hacia las demandas y expectativas de partes interesadas ajenas a la comunidad universitaria, ganancias de productividad académica, generación de excedentes, contribución al desarrollo regional y local, internacionalización, etc.

Vale resaltar que, a lo largo de todos estos años, las universidades están pasando por un proceso de innovación por medio de acreditaciones internacionales, con el fin de adaptarse a todas las necesidades que diario se presentan y así poder competir con universidades no solo locales, sino, internacionales. Este libro aborda puntos muy bien estructurados de cómo ha sido la evolución de los actores han influido en los cambios de las políticas públicas y universitarias.

Las organizaciones internacionales o supranacionales han ido ganando influencia en las políticas para la educación superior en el mundo. Cada vez se las universidades crecen con las influencias de estas organizaciones internacionales, para poder tener un mejor nivel universitario, considerando sus influencias e impacto social y así, provocar una internacionalización de las políticas universitarias.

Cuestiones de competitividad y problemas de escasez de fondos, llevaron a nuevas exigencias de especialización y creación de perfiles líderes, igualmente, no todas las universidades deberían seguir ofertando un amplio espectro de programas y servicios donde tengan lagunas y no se pueda implementar correctamente, es preferible tener un programa investigador variado. La especialización y el esfuerzo en establecer un perfil posiblemente único podría ser una solución a la escasez de fondos y a la alta competitividad. La búsqueda de la excelencia o primeros puestos en rankings nacionales o internacionales ha provocado una mayor participación de los sistemas universitarios nacionales. Las consecuencias se pueden ver en el surgimiento de nuevos subsistemas y redes que tienen cierto impacto en la lógica de los sistemas universitarios.

Para sobrellevar las nuevas responsabilidades en su gestión, las universidades han creado nuevos puestos de trabajo en todas las áreas de la universidad, así como de gestión o han delegado más responsabilidades estratégicas a los trabajos administrativos, esto con el fin de crear un ambiente más competitivo y poder alcanzar un mejor lugar en la tabla de posicionamientos.

Los nuevos profesionales en la universidad, producto de estos cambios, suelen estar altamente cualificados y llevan a cabo tareas determinantes tanto en los procesos de docencia e investigación como de gestión al más alto nivel. Preparan y mantienen asuntos de alto nivel, ofrecen servicios o desempeñan funciones que hacen de puente entre las unidades básicas y la dirección central. De este modo, los objetivos de las universidades en todos los niveles están identificados mediante actividades de gestión con el fin de aumentar la eficiencia y la efectividad.

Aún queda mucho que hacer para reforzar todas estas áreas de competitividad, una herramienta que ayuda a mejorar las relaciones entre universidades internacionales es crear pactos entre ellas para implementar los intercambios académicos, las capacitaciones, mejorar la calidad educativa, entre muchas otras acciones. El número de acciones con las que las reformas internas de las estructuras en las universidades se

Revista Política, Globalidad y Ciudadanía, Vol. 4 No. 8, Julio - Diciembre 2018, Universidad Autónoma de Nuevo León, Monterrey, México, ISSN 2395-8448. pp 14-19. http://revpoliticas.uanl.mx/index.php/RPGyC/article/view/99 
implementan es muy alto, esto quiere decir que, al implementar nuevos puestos y la mejora de un salario, los docentes aumentan su rendimiento en el trabajo para lograr un mayor desempeño.

La introducción de reformas inspiradas en la nueva gestión pública en las universidades tiene como objetivo alcanzar una doble transformación, la combinación de instrumentos varía según el país o la institución. Se debe de realizar una combinación de todos los recursos que tengan para poder realizar reformas exitosas e implementarlas correctamente.

El libro también toca unos puntos muy importantes, sobre como las dificultades e inconvenientes que han tenido que superar para conseguir un determinado objetivo y mejorar en todos los aspectos las ramas de la educación.

El ámbito de la gestión universitaria, el día a día, trae consigo una serie de situaciones que resultan complicadas y dificultan la labor del gestor. Actualmente en la sociedad, cada día, los gobiernos tienen la responsabilidad de generar cambios y mejoras en las instituciones, imponer nuevos retos educativos, para poder realizar nuevos modelos educativos.

\section{3.- MÉTODO}

Diseño

El enfoque investigativo de la presente investigación es cualitativo, de acuerdo con Hernández, Batista y Fernández (2014) "Utiliza la recolección y análisis de los datos para afinar las preguntas de investigación o revelar nuevas interrogantes en el proceso de interpretación” (p.7).

Alcanzando un diseño no experimental "Que se realiza sin la manipulación deliberada de variables y en los que sólo se observan los fenómenos en su ambiente natural para después analizarlos" (Hernández, Batista y Fernández, 2014, p. 149).

El alcance establecido es el exploratorio "emplean cuando el objetivo consiste en examinar un tema poco estudiado o novedoso" (Hernández, Batista y Fernández, 2014, p. 91).

Instrumentos

Para la construcción del marco teórico-conceptual de la nueva gobernanza de los sistemas universitarios, se consultaron un total de ocho referencias bibliográficas utilizándose como instrumento las ideas, argumentos y proyectos que fueron interpretados desde una perspectiva analítica y crítica.

Procedimiento

Con relación a la comprensión del problema de la investigación se recopilan fuentes secundarias de documentos académicos. En el marco de referencia se definen los conceptos básicos relativos a la nueva gobernanza de los sistemas universitarios. Una vez recopilada y analizada la información se construye el documento objeto de este trabajo. Por último, se realizan las recomendaciones y conclusiones conforme a los objetivos trazados (Oliveros et al, 2018, p. 72).

\section{4.- CONCLUSIONES}

Finalmente, se concluye que las políticas educativas responden cada vez más a una arena internacional en la que están sucediendo la configuración de agendas, los desarrollos de criterios y directrices, el establecimiento de objetivos y la clasificación más resistentes y difíciles de modificar son las ideologías emanadas de cada uno de los regímenes de gobernanza universitaria y de sus principales elementos que los dispositivos organizacionales, de economía política y jurídica que los acompañan, que estos hacen que la gestión universitaria tenga sus fortalezas y sus debilidades. 
Lo que estos desarrollos educativos para los gobiernos y las autoridades necesitan saber que las responsabilidades y las políticas sobre la gobernanza en la educación superior en un nivel sistémico han dejado de ser una responsabilidad exclusiva de los gobiernos nacionales. Algunas responsabilidades se han trasladado hacia arriba, a niveles supranacionales; otras, hacia abajo, al nivel institucional a través de una desregulación y otras, hacia fuera, a agencias dependientes o semiindependientes.

Esta es una gran tarea que todos tenemos y que aún no se ha podido completar, se necesitan realizar políticas públicas de alto impacto para poder mejorar las políticas universitarias, también los cambios que se han realizado por las reformas educativas en la gobernanza han hecho a las universidades pasar de instituciones administradoras a organizaciones, las relaciones entre las universidades y el gobierno público han sido redefinidas y basadas en nuevas ideas sobre qué debe ser dirigido y cómo.

Así mismo, la idea de generar nuevos líderes universitarios y políticos, para poder tener una mayor participación directa e indirectamente es muy importante, ya que son actores en esta nueva etapa universitaria.

Este artículo establece por puntos una comparación de la gobernanza universitaria en Europa con otros países de Latinoamérica, donde se habla que aun que en cada país tenga su propio avance en estas áreas, aún queda mucho por hacer de la mano con el gobierno y sus reformas educativas.

\section{REFERENCIAS}

Acosta, A. (2008). La autonomía universitaria en América Latina: Problemas, desafíos y temas capitales. Universidades.

González García, Y. (2008). El reto de la autonomía universitaria. Universidades, Año LVIII.

Hernández, R., Fernández, C., y Baptista, P. (2014). Metodología de la investigación. México: Mc Graw Hill.

Meléndez Guerrero, M. A., Solís Pérez, P. C. y Gómez Romero, J. G. I. (2010). Gobernanza y gestión de la universidad pública. Revista de Ciencias Sociales (RCS), XVI, 2, 210-225

Oliveros, O.; Waked, D.; Peña, C. y Lechuga, J. (2017). Diagnóstico Sobre la Planeación del Mercadeo en las Pymes de Boyacá. Revista Desarrollo Gerencial, 9 (2), 68- 87.

Rubio, F. (2012). Innovación y buenas prácticas en el gobierno y la gestión de universidades. Revista de Universidad y Sociedad del Conocimiento (RUSC).

Schumpeter, J. (1978). Teoría del desenvolvimiento económico. México: Fondo de Cultura Económica. Quinta Reimpresión.

Schwartzman, S. (1996). El papel de la universidad en el desarrollo social. Revista de Educación Superior, $30(1), 117,99-104$. 